\title{
Respiratory Rehabilitation in the Covid-19 Patient Care Journey: Summary of International Recommendations
}

\author{
F. El Abed* and F. Benlebna \\ Department of Physical Medicine and Rehabilitation, EHU Oran, Algeria
}

\begin{abstract}
Respiratory disorders are at the fore front of severe COVID-19 disease and can lead to acute respiratory distress syndrome (ARDS). This article provides a few international recommendations for the practice of respiratory rehabilitation in patients infected with the SARS-CoV-2 virus.

We report a summary of national and international recommendations published before October 11,2020 , concerning respiratory rehabilitation in the management of COVID + patients.

Various recommendations were retained, relating to the evaluation criteria, the indications for respiratory rehabilitation and the methods of treatment along the course of treatment.

Respiratory rehabilitation is essential in the care process for COVID + patients and in compliance with the rules of good practice necessary to limit the risks. In this context, the recommendations represent a guide to improve the management of respiratory disorders in daily practice, as well as the quality of life of patients.
\end{abstract}

\section{Keywords}

Respiratory rehabilitation, Recommendations, Care pathway, Quality of life, COVID-19

\section{Introduction}

The global health crisis, which has now persisted for a year, is due to an attack by the SARS-CoV-2 coronavirus. It is a highly contagious human-to-human viral infection associated with significant mortality, especially in the elderly.

Since the start of the pandemic, knowledge about the disease has become more and more precise. COVID-19 is responsible for respiratory damage, but also for cardiovascular, neuro-cognitive, digestive, renal, metabolic, psychiatric, etc. impairments.

In $80 \%$ of cases, people infected with the SARS-CoV-2 virus are paucisymptomatic or asymptomatic, while $20 \%$ present with severe symptoms requiring hospitalization. Apart from age, male sex, obesity, a history of high blood pressure, diabetes or other cardiovascular diseases seem to be risk factors for severe forms [1,2].

In cases of severe COVID-19 (15\%), major respiratory disorders are often found with acute respiratory distress syndrome (ARDS), which requires respiratory assistance (5\%) [1]. Pulmonary fibrosis is one of the early complications of this syndrome, which can be aggravated by mechanical ventilation. There is evidence for early fibrotic changes in ARDS, $47 \%$ of patients have radiologic signs of pulmonary fibrosis from day one [3].
Even after a short period of ventilation, patients have been observed to exhibit disturbances in peripheral oxygen saturation $\left(\mathrm{SpO}_{2}\right)$ and pathological chest imaging for several weeks after infection. According to some authors these abnormalities can persist for several years after the acute phase [4].

The possible sequelae of the disease are due to the specific attacks of the viral infection and the runaway immune system. They are also secondary to the "nonspecific" complications of ARDS, immobility and prolonged hospitalizations in intensive care.

During the stay in the intensive care unit, immobilization can result in the appearance of pressure ulcers, musculotendinous contractures, intensive care neuromyopathy, pulmonary

*Corresponding author: F. El Abed, Department of Physical Medicine and Rehabilitation, EHU Oran, Algeria

Accepted: June 12, 2021

Published online: June 14, 2021

Citation: Abed FE, Benlebna F (2021) Respiratory Rehabilitation in the Covid-19 Patient Care Journey: Summary of International Recommendations. Res Rev Infect Dis 4(1):138-144 
fibrosis, etc. Various studies have demonstrated the benefit of early rehabilitation treatment [3].

In the aftermath of the disease, patients present with significant asthenia, exercise deconditioning, persistent respiratory disorders, reduction, or even loss of autonomy, which require interdisciplinary management in physical medicine and rehabilitation (MPR) to improve recovery and shorten the time to return to normal life.

The objective of this article is to provide a scientific update and a pragmatic description of the diagnostic and therapeutic attitude adopted in physical medicine and rehabilitation in the essentially respiratory care pathway of the COVID-19 patient.

\section{Methodology}

We report a summary of internationals recommendations concerning respiratory rehabilitation in the management of COVID-19 + patients published before October 11, 2020; namely the WHO 2020 recommendations, as well as Chinese, European and American recommendations.

The searches were carried out until October 11, 2020 on Google scholar, using some keywords: Respiratory rehabilitation, Covid-19, care pathway, recommendations, and quality of life. We selected the articles with respiratory rehabilitation at the different stages of management of the Covid-19 disease, namely during the hospitalization of patients and after discharge from the hospital. The quality assessment was carried out independently, and we have adopted the recommendations of the learned societies that are usually consulted and WHO recommendations since this supposes summarizing the different recommendations around the world.

\section{Principles, Concept and Characteristics of Respiratory Rehabilitation: Recommendations}

Recommendations for respiratory rehabilitation in COVID-19 are established by various learned societies and scientific medical teams around the world:

\section{According to Swiss recommendation}

The rehabilitation program must take into account the multisystem aspect of the disease as well as its course. The evaluation and follow-up criteria will make it possible to improve care and also to better understand the evolution of patients. The clinical criteria used must be simple and reproducible.

Among the clinical scores used, respiratory rate, peripheral oxygen saturation $\left(\mathrm{SpO}_{2}\right)$, the Borg scale and the 6-minute walk test, have the advantage of being reproducible, easy to perform and a good reflection of the endurance on effort.

Respiratory rehabilitation must especially emphasize ventilation techniques and postural drainage in the event of bronchial congestion, on gradual rehabilitation during exercise. The objective is to achieve an $\mathrm{SpO}_{2}$ equivalent or greater than $92 \%$ in air ambient and stable after stress [3].
Rehabilitation is similar to that of an ARDS for patients who have stayed in intensive care, and to that of a deconditioned patient for less severe patients, with two particularities:

- The strong contagiousness of the viral infection which requires maintaining the protective measures until a multidisciplinary decision to lift the isolation involving infectious disease specialists;

- The fragility of these patients likely to present sudden medical decompensations such as respiratory failure, thromboembolic or cardiovascular complications.

Rehabilitation professionals must act according to the individual needs of each patient with exercise reconditioning in the chronic phase. The intervention of psychologists is essential.

The monitoring of physiological constants at rest and during exercise, in particular respiratory, must be reinforced throughout the duration of the rehabilitation: Respiratory rate and oxygen saturation, heart rate. The practice of a stress ECG during the first sessions of rehabilitation may be useful for some patients depending on their comorbidities.

\section{According to Chinese recommendations}

From a physiotherapy maneuver point of view, nothing new, working the respiratory muscles, learning to control sputum, general gymnastics, all of this is well known. What is more original are the conditions under which they must be applied [5].

1. The ventilation of the room: A ventilation system must be installed before the arrival of the patient: Ventilator pulsed towards the patient at the entrance of the room, ventilator pulsed towards the outside, on the other side of the patient.

2. Healthcare personnel should wear protective clothing, medical mask, protective face shield or eye protection.

3. When possible, respiratory rehabilitation should be done by intercom behind a glass wall. Otherwise, the therapist should never pass downwind, that is, between the patient and the extractor ventilation system.

4. Avoid contact with the patient.

5. For coughing, expectoration or sneezing exercises: the patient faces the exhaust fan towards the window.

6. When the patient coughs, spits and sneezes, a tissue should be placed around the mouth and nose before coughing, spitting and sneezing; soiled fabrics should be kept in a covered waste bin.

7. Disinfection is done twice a day on all surfaces using chlorinated products.

8. Two decluttering sessions should be done every day, plus one gymnastics session (bridging, aerial pedaling, lower and upper limb work), including those with hypoxemia (add oxygen during exercises).

In summary, this recommendation involves adopting extremely cautious behavior to limit the risk of contamination. 


\section{According to WHO May 2020 recommendation}

"When rehabilitation needs are identified, refer the person for follow-up in a hospital setting, in outpatient clinics or in the community, according to the indications and needs" [6]:

1. Ensure that appropriate infection control measures are available in designated rehabilitation areas that manage patients with still contagious COVID-19.

2. When a patient no longer requires an intensive care bed, but needs rehabilitation in a hospital setting, refer him to a rehabilitation service or an institution that can provide the required level of care.

3. When a patient does not require in-hospital rehabilitation, but would benefit from follow-up and rehabilitation after discharge, refer them to an outpatient or community care service depending on local availability. Ensure patients receive the education and information necessary for selfmanagement.

"Offer personalized rehabilitation programs ranging from post-acute to long-term care, according to the patient's needs" [6]:

1. Guarantee access to multidisciplinary rehabilitation where patients will have access to professionals whose skills correspond to their needs. This can include physical therapists, occupational therapists, speech language pathologists, mental and psychosocial health providers and, in complex cases, physical medicine and rehabilitation specialists. However, the composition of rehabilitation staff may vary depending on the context and in different parts of the world.

2. Rehabilitation programs should be focused on the patient's needs and goals and may include exercise, self-management education and counseling, breathing techniques, support and education for caregivers, support groups, stress management and home adaptation.

3. Supplement rehabilitation with educational resources, such as information brochures on expected symptoms, exercise and self-management, as well as advice for caregivers.

4. When long-term rehabilitation needs are evident due to severe respiratory disease and post-resuscitation syndrome, for example in cases of persistent fatigue, reduced exercise tolerance and difficulty in carrying out in day-to-day activities, patients can benefit from pulmonary rehabilitation programs (or equivalent) within the community.

\section{According to French recommendations}

Rapid responses have been developed collegially between the Haute Autorité de Santé (HAS) and the French Society of Physical Medicine and Rehabilitation (SOFMER).

These are, on the one hand, responses aimed at promoting the organization of the journey of COVID + patients leaving the Medicine, Surgery, Obstetrics (MCO) or intensive care units, to the home, or to the Aftercare units. and Rehabilitation
(SSR) before their return home [7] (Table 1); and on the other hand, rapid responses within the framework of the management of post-COVID-19 patients in Physical Medicine and Rehabilitation (MPR), in Aftercare and Rehabilitation (SSR) and return home (Table 2 ) [8].

Rehabilitation in intensive care units: Patients with the most severe forms of Covid-19 are hospitalized in intensive care in order to maintain their vital functions, especially breathing. At this point, the challenge is to prevent and limit complications once released from intensive care. This is particularly important for these patients who are at risk of presenting numerous sequelae, in particular respiratory.

Treatment must begin as soon as possible, in collaboration with the resuscitators. The HAS [7] specifies that "this support is adapted to the initial assessment which assesses the patient's failures and deficiencies. Its clinical state, state of consciousness and alertness, severity of respiratory failure, ventilation mode and the importance of life support techniques are taken into account".

She details the possible interventions: "Respiratory rehabilitation, for example, makes it possible to improve the quality of ventilation and facilitates respiratory weaning, that is to say the patient's ability to relearn to breathe on his own once he is ex. cased" [7].

It also describes the rehabilitation/rehabilitation sessions according to the patient's state of consciousness. "In sedated or unconscious patients, passive mobilizations and limb postures aim to limit loss of range of motion and cutaneous complications. Conscious patients (ventilated or not), on the other hand, should benefit from rehabilitation/rehabilitation interventions that include active muscle exercises, exercise cardiorespiratory reconditioning, changes in posture while sitting or standing upright and preparation for the resumption of functional activities" [7].

Rehabilitation in Post Resuscitation Rehabilitation Care (SRPR) units: On leaving intensive care, it is necessary to structure units whose mission is to wean off mechanical ventilation and provide early rehabilitation care for patients, in a context of limited beds and human resources allocated to intensive care.

They constitute a bridge between the intensive care services and the specialized rehabilitation care services. They receive patients from intensive care units who require medical supervision with specific care. They offer early rehabilitation, as well as specialized and multidisciplinary medical follow-up. Management should allow patients to regain autonomy and respiratory independence.

The SRPRs will take care of patients with major respiratory failure, intubated or not, still dependent on equipment (tracheostomy, non-invasive ventilation, invasive ventilation). These SRPRs will help reduce the length of stay for patients in intensive care. The "Pneumological SRPRs" are intended to take care of patients with a unique deficiency in respiratory function. The management objectives are respiratory weaning, exercise reconditioning and respiratory rehabilitation [9]. 
Table 1: Rapid responses in the context of COVID-19 - Rehabilitation journey of the COVID + patient upon discharge from intensive care and/ or OLS, in SRH then at home [7].

\begin{tabular}{|c|c|}
\hline \multicolumn{2}{|c|}{ Conditions of transfer: } \\
\hline Quick response $n^{\circ} 1$ & $\begin{array}{l}\text { - No direct admission to SSR from intensive care. } \\
\text { - Criteria allowing the transfer of a COVID + patient from a "Medicine, Surgery, Obstetrics" (MCO) service to a } \\
\text { Aftercare and Rehabilitation Unit (SSR): } \\
\text { - A patient can be admitted to a COVID-19 SSR unit: } \\
\text { o From the } 10^{\text {th }} \text { day after the onset of symptoms or entry into intensive care, if he has presented a severe form, } \\
\text { o From the } 7^{\text {th }} \text { day after the onset of symptoms after a stay in OLS alone for a non-serious form, } \\
\text { o If possible at least } 48 \text { hours after improvement of symptoms: reduction of dyspnea, respiratory rate }<22 / \\
\text { min, temperature }<37.8^{\circ} \mathrm{C} \text {, oxygen }\left(\mathrm{O}_{2}\right) \text { requirements stabilized, hemodynamic stability. } \\
\text { - Prefer an SSR near a short stay if transfer }<\text { D7 or }<\text { D10 after the onset of symptoms (possible aggravation } \\
\text { between D6 and D9). } \\
\text { - Social problems, without being neglected, do not in themselves constitute a sufficient reason for addressing } \\
\text { SSR, other avenues must therefore be sought. }\end{array}$ \\
\hline \multicolumn{2}{|c|}{ Objectives of the SSR stay: } \\
\hline Quick response $n^{\circ} 2$ & $\begin{array}{l}\text { The rehabilitation needs of most COVID patients are related to exercise re-training, respiratory rehabilitation, } \\
\text { overall muscle strengthening, re-empowerment in activities of daily living, treatment of psychomotor } \\
\text { disorders, treatment possible complications, with the installation of: } \\
\text { HAS - Rapid responses in the context of COVID-19 - Rehabilitation pathway for the COVID + patient on } \\
\text { discharge from intensive care and/or OLS, in SSR and then at home } \bullet \text { June } 2020 \\
\text { An adapted therapeutic education program, rehabilitation of perceptual-motor disorders, nutritional } \\
\text { monitoring, and psychological support. }\end{array}$ \\
\hline Quick response $n^{\circ} 3$ & $\begin{array}{l}\text { For rehabilitation and rehabilitation activities, the benefit/risk ratio must be assessed individually, prioritizing } \\
\text { activities whose postponement would lead to a loss of opportunity for the patient or worsening of a comorbidity. }\end{array}$ \\
\hline \multicolumn{2}{|l|}{ Reception conditions: } \\
\hline Quick response $n^{\circ} 4$ & $\begin{array}{l}\text { The COVID SSR unit must be equipped to meet the needs of the patient. An organization adapted to the risk } \\
\text { of the virus circulating within the establishment must be respected. Access to the technical platform must be } \\
\text { planned in order to offer different time slots for COVID patients in the post-isolation period (between D10 } \\
\text { and D24 after the onset of symptoms) and COVID "cured on the infectious level" (> D } 24 \text { after the onset of } \\
\text { symptoms and > } 14 \text { days after discharge from intensive care). }\end{array}$ \\
\hline Quick response $n^{\circ} 5$ & $\begin{array}{l}\text { Criterion allowing return home, regardless of the type of follow-up: Return to the functional level and to the } \\
\text { previous state of autonomy, or approaching if sufficient home help can be organized upstream of the return. } \\
\text { The patient's return home requires their active participation and often that of those around them. }\end{array}$ \\
\hline
\end{tabular}

Table 2: Rapid responses in the context of COVID-19 - Management of post-COVID-19 patients in Physical Medicine and Rehabilitation (MPR) [8].

The more or less severe deficiencies of respiratory, cardiovascular, renal, neurocognitive, psychiatric,

Quick response $n^{\circ} \mathbf{1}$ musculoskeletal, metabolic and nutritional orders, leading to a limitation of activity, are frequent and particularly important in these patients, and will require taking in prolonged charge.

Quick response $n^{\circ} 2$ The risk of contamination requires strict application of protective measures during rehabilitation/rehabilitation sessions during the contagious phase which can last beyond the acute phase.

The objectives of PRM hospitalization are the diagnostic workup and assessment of specific impairments and activity

Quick response $n^{\circ} 3$ limitations, the structuring of rehabilitation/rehabilitation programs, and the monitoring of medical complications.

Quick response $n^{\circ} 4$ Some patients require a multi-professional rehabilitation/rehabilitation program coordinated by a doctor from MPR. The use of oxygen therapy is often necessary.

As long as the patient is not stabilized, rehabilitation/rehabilitation must take into account the risk of

Quick response $n^{\circ} 5$ cardiorespiratory decompensation and specific thromboembolic complications, with monitoring of physiological constants.

Quick response ${ }^{\circ} 6$

Each rehabilitation/rehabilitation intervention must take into account the fatigability of these patients who are often undernourished, asthenic and carry co-morbidities.

Quick response $n^{\circ} 7$

Rehabilitation at home can be carried out by tele-care, or with exercise self-programs previously learned and supervised remotely, or by a physiotherapist at home if his absence causes a loss of luck for the patient.

To constitute an SRPR unit, establishments must meet the following requirements [9]:

- Equipment: Respirators; Monitoring devices (heart rate/arterial pressure/oxygen saturation); Nasogastric tube; Emergency trolley; Bronchial fibroscopy available 24 hours a day; Chest X-rays in bed 24 hours a day.

- On-site medical care, $24 / 7$, potentially shared with other areas of the establishment.
- Doctors: From intensive medicine, intensive care or anesthesia-intensive care and a pulmonologist and/or doctor specializing in physical and rehabilitation medicine, in all cases trained in mechanical ventilation and emergency situations.

- Paramedical teams made up of: Nurses and caregivers trained in respiratory resuscitation and the devices used, physiotherapists trained in motor and respiratory care, occupational therapists, speech therapists trained in the 
care of tracheostomy patients and disorders of swallowing, dietitian, psychologist.

- Quick, local, early and coordinated access to an adapted intensive care or intensive care unit.

Rehabilitation in physical medicine and rehabilitation hospital units: Patients coming out of intensive care or continuing care are for the most part referred to PRM units specializing in respiratory ailments. These are units allowing the care of patients who cannot return directly to their homes, without the need for complex care and without respiratory assistance. However, these units can take care of patients requiring non-complex respiratory assistance (oxygen supply, excluding high flow), provided they meet the following requirements:

- Availability of fluids in the room allowing the establishment of oxygen therapy;

- Operational medical on-call, 24/7, with medical availability on site;

- Paramedical teams made up of: Masseur-physiotherapist, dietitian, psychologist, personnel skilled in the handling of oxygen therapy equipment.

Before prescribing rehabilitation, a clinical evaluation is necessary, namely:

- Report the date of onset of symptoms, the criteria for the severity of the history of acute infectious lung disease, search for a history of respiratory disease, other possible COVID-19 damage and the duration of mechanical ventilation.

- Respiratory rate, dyspnea, transcutaneous oxygen saturation at rest and after exercise.

- Appreciate the effort tolerance by the modified Borg scale. The latter reports the evolution of dyspnea during exercise of increasing intensity from 0 to 10 , thus making it possible to deduce the burden of respiratory rehabilitation (Figure 1) [10].

Measure functional capacities for daily activities using the Barthel scale. It is one of the most widely used indices in physical medicine and rehabilitation. It includes ten items analyzing patients' autonomy in activities of daily living: Food, personal care, bathing, dressing, urinary continence, fecal

\begin{tabular}{|c|l|}
\hline Intensity of effort & Dyspnea on effort \\
\hline $\mathbf{1 0}$ & Maximum shortness of breath \\
\hline $\mathbf{9}$ & Extremely severe shortness of breath \\
\hline $\mathbf{8}$ & Very severe shortness of breath \\
\hline $\mathbf{6}$ & \\
\hline $\mathbf{5}$ & Severe shortness of breath \\
\hline $\mathbf{4}$ & Almost severe shortness of breath \\
\hline $\mathbf{3}$ & Moderate shortness of breath \\
\hline $\mathbf{2}$ & Mild shortness of breath \\
\hline $\mathbf{1}$ & Very slight shortness of breath \\
\hline $\mathbf{0 . 5}$ & Barely noticeable shortness of breath \\
\hline $\mathbf{0}$ & No shortness of breath \\
\hline
\end{tabular}

Figure 1: Modified borg scale [10]. continence, toilet use, bed-chair transfers, walking and stairs. Each item is rated $0,5,10$, or even 15 for certain items. The maximum score is $100(100=$ complete independence, $0=$ total dependence).

- 10-meter and 6-minute walk tests, descent/staircase test.

- Exercise tolerance: maximum/submaximal exercise tests (ergometers adapted to the patient's abilities, stepper test with monitoring of oxygen saturation $\left(\mathrm{O}_{2}\right)$ during exercise).

Depending on the indication, request an exercise ECG, $\mathrm{VO}_{2}$-max with blood gas analysis. But also the Peak flow, measuring the maximum inspiratory and expiratory pressures, as well as the respiratory functional explorations (FR).

During the respiratory rehabilitation program, a few instructions must be observed:

- Ventilatory exercises may possibly be offered to a stable patient who is not polypneic at rest (Respiratory Rate $\leq 22$ ) to prepare for reconditioning to efforts (muscle strengthening and adapted overall endurance work) which has shown its effectiveness.

- The proposed respiratory exercises are implemented according to the deficiencies of the respiratory muscles, the expiratory capacities and the cough.

- Exercises aimed at improving ventilation control are performed without direct supervision with the objectives of increasing tidal volume, reducing psychological consequences (stress, anxiety and depression), with an expiratory time longer than the inspiratory time in order to 'avoid diaphragmatic fatigability [8].

- Favor self-rehabilitation on verbal prompting, ventilate the room before the caregivers access it.

- Respiratory physiotherapy techniques for bronchial drainage are used with caution. During this phase, education and self-rehabilitation are privileged. If ventilatory capacities are impaired, offer instrumented drainage.

- The pursed-lip work used during exertion has shown an effect on respiratory rate and minute ventilation in people with COPD, but we do not know the degree of obstructive involvement in COVID-19. The pursed-lip work would have an impact on gasometric parameters at rest through its positive expiratory pressure effect, but these data are old. However, because COVID-19 patients probably have weakness in their expiratory muscles. Respiratory work with expiratory brake retains indications in this population. It may be poorly tolerated in terms of respiratory fatigue and circulatory tolerance [8].

- When any of the following conditions occur during rehabilitation, the MPR doctor should immediately stop the exercise: resting polypnea $>22 / \mathrm{min}$, chest tightness and/or pain, shortness of breath, malaise, headache, blurred vision, palpitations, sweating, cyanosis, clouding, confusion, inability to speak, sign of diaphragmatic dysfunction (antepnea, thoraco-abdominal asynchrony, paradoxical breathing), etc. 
- Decrease in $\mathrm{SpO}_{2}>4$ points, compared to resting $\mathrm{SpO}_{2}$, requires adjustment in exercise intensity and $\mathrm{O}_{2}$ administration. The monitoring of exercise tolerance is to be performed by modified Borg scale, pulse measurement, and $\mathrm{SpO}_{2}[8]$.

At the same time, a nutritional caloric and protein monitoring must be systematic given the increase in energy expenditure of ventilatory work, but also the presence of anorexia secondary to the infection, stress, and the syndrome of inappropriate refeeding [8]. Psychotherapy is offered for anxiety-depression such as post-traumatic stress disorder. Support for resuming lifestyle habits and social participation is necessary, with remote monitoring of rehabilitation in the event of sequelae reducing autonomy.

Home rehabilitation: Home rehabilitation is organized in different ways: liberal professionals at home, tele-care, mobile team. The role of the attending physician is essential. An MPR opinion for an exercise reconditioning or socio-professional reintegration program can be offered in agreement with the attending physician. Occupational therapists can assess the home, if necessary, to make it as secure as possible. Pneumological and cardiological monitoring is systematically offered to patients remotely. Outpatient activities have a socio-family interest for the patient, but also help to avoid overcrowding in full hospitalization services. Two clinical situations can be seen; these are:

- Or post-COVID-19 patients discharged at home following hospital treatment: Rehabilitation must be adapted to any pulmonary deficiencies. It should also help the resumption of light physical activity with respect for fatigue given the importance of the latter in post covid. Renutrition started during hospitalization should be continued and monitored. The help of a dietician or a nutritionist may be requested. Face-to-face or tele-care psychological follow-up if necessary can be offered at home as a continuation of inpatient care.

- Or COVID-19 patients, with no indication for hospitalization, confined and monitored at home: In the acute phase and during the symptomatic phase of the disease, physical activities are contraindicated. At this phase, respiratory rehabilitation is not systematic with bronchial clearance if there is superinfection, while respecting the rules of protection of the caregiver. Once the acute phase has passed, an adapted respiratory rehabilitation is offered according to the clinical signs, while respecting the patient's fatigue, dyspnea and in the absence of fever. The help of a dietician or a nutritionist may be requested for appropriate refeeding. Psychological support should be offered depending on the situation.

Respiratory rehabilitation on an outpatient basis must comply with safety measures, according to different situations (Day hospitalization; Mobile Rehabilitation-Reintegration teams; Outpatient consultations; Rehabilitation home hospitalization; Coverage by private rehabilitators in the office, at home and/or by tele-care). Whatever the situation, the benefit/risk ratio must be assessed each time individually, prioritizing the most urgent activities. In addition, an organization adapted to the risk of the virus circulating within the establishment during patient visits should be respected, namely:

- Avoid or limit the number of accompanying persons (only one maximum per patient).

- Ensure compliance with barrier measures by medical transporters.

- Organize flows to prevent incoming and outgoing patients from crossing paths.

Global exercise reconditioning is targeted at cardiorespiratory and muscular endurance, in day hospitalization or in town, to prepare for the return to work (vocational rehabilitation) and physical and social activities.

Figure 2 summarizes the respiratory rehabilitation recommendations throughout the care pathway for COVID + patients.

\section{According to European and American recom- mendations}

European Respiratory Society and American Thoracic Society recommends early, bedside rehabilitation for patients affected by severe COVID-19. The model of pulmonary rehabilitation may suit as a framework, particularly in a subset of patients with long-term respiratory consequences [11].

Rehabilitation societies including the Turkish Society of Physical Medicine and Rehabilitation have issued pulmonary rehability recommendations in COVID-19 pneumonia with productive cough can benefit from diaphragmatic breathing, pursed-lip breathing, and resistance-breathing training. Besides, those in mechanical ventilation and post-intensive care syndrome COVID-19 cases, oxygen therapy, early mobilization, airway clearance, aerobic exercise, gradualgraded limb muscle resistance exercise, nutritional and psychological interventions should be consideration. During pulmonary rehabilitation, careful evaluation of vital signs and exercise-induced symptoms is also required. When in-person pulmonary rehabilitation is not possible, tele rehabilitation should be explored. However, the long-term effects of PR in COVID-19 need further evaluation [12].

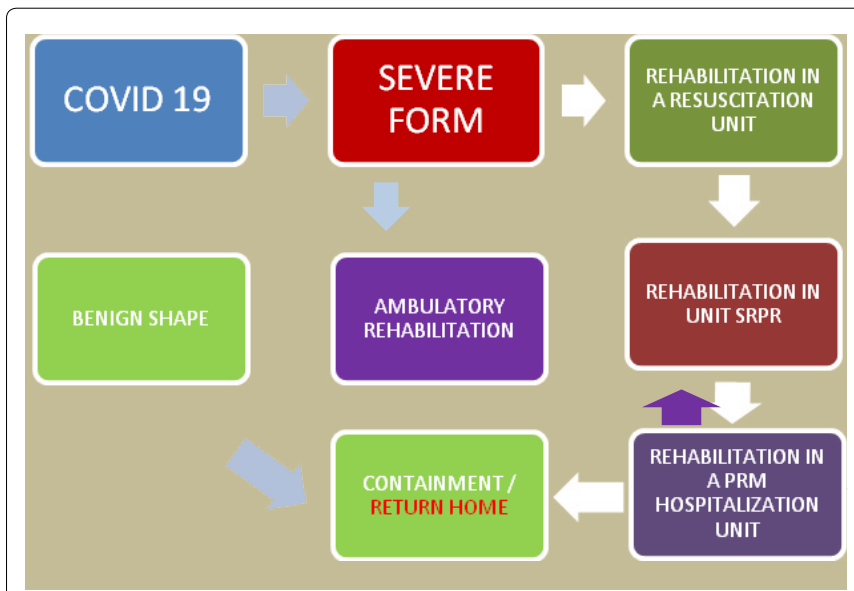

Figure 2: Respiratory rehabilitation circuit in the management of COVID-19 according to French recommendations.

SRPR: Post-resuscitation rehabilitation care; MPR: Physical and rehabilitation medicine. 
Summary of analysis-perspective of Wang and al. report that referring patients for rehabilitation should be judged case-case, based-on discussion with other professionals in the multidisciplinary team, less time consuming than before because of patient over load at emergency, rehabilitation specialist now is considered an integral part of a treating team, therapy period should also be readjusted [13].

\section{Conclusion}

Respiratory rehabilitation must be undertaken at each stage of the disease Covid; thus, in the acute phase, it must above all insist on ventilation techniques and on postural drainage in the event of bronchial obstruction. In the subacute and chronic phases, respiratory rehabilitation, in addition to the techniques of the acute phase, will emphasize progressive rehabilitation to exercise with the objective of achieving an $\mathrm{SpO}_{2}$ equivalent or greater than $92 \%$ in ambient air and stable after effort.

Otherwise in view of the evolution of the COVID-19 epidemic, the creation of organized networks and care pathways in rehabilitation, particularly respiratory care, are levers to be activated as soon as possible to provide the medium and long-term care necessary for the acquisition of a better quality of life for patients.

\section{References}

1. www.who.int/docs/default-source/coronaviruse/situationreports/20200306-sitrep-46-covid-19.pdf?sfvrsn=96b04adf_4

2. www.ecdc.europa.eu/sites/default/files/documents/covid19-rapid-risk-assessment-coronavirus-disease-2019-eighthupdate-8-april-2020.pdf

3. Grácio S, Koçer S (2020) Rehabilitation: Essential for survivors of severe COVID-19. Swiss Medical Review.
4. Zhang P, Li J, Liu H, et al. (2020) Long-term bone and lung consequences associated with hospital-acquired severe acute respiratory syndrome: A 15 -year follow-up from a prospective cohort study. Bone Research 8: 8.

5. Yang F, Liu N, Hu JY, et al. (2020) Pulmonary rehabilitation guidelines in the principle of 4S for patients infected with 2019 Novel Coronavirus (2019-nCoV) 43: 180-182.

6. (2020) Clinical management of COVID-19 Provisional guidance. World Health Organization.

7. (2020) Haute Autorité de Santé (HAS), French Society for Physical Medicine and Rehabilitation (SOFMER). Rapid responses in the context of COVID-19 - Rehabilitation journey of the COVID + patient upon discharge from intensive care and/or OLS, in SSR and then at home.

8. (2020) Haute Autorité de Santé (HAS), French Society for Physical Medicine and Rehabilitation (SOFMER). Rapid responses in the context of COVID-19 - Management of post-COVID-19 patients in Physical Medicine and Rehabilitation (MPR), in Aftercare and Rehabilitation (SSR) and return home, rapid response method.

9. (2020) Ssr Establishments And Services Sheet; Recommendations for Structuring Ssr Support for Covid + Patients Exiting Short Stay. French Ministry of Solidarity and Health.

10. Selleron B (2014) Measuring breathlessness in 4 dimensions. Focus Cardio-Respiratory Physiotherapy.

11. Spruit MA, Holland AE, Singh SJ, et al. (2020) COVID-19: Interim guidance on rehabilitation in the hospital and post-hospital phase from a European Respiratory Society- and American Thoracic Society-coordinated international task force. Eur Respir J 56: 2002197.

12. Siddiq MAB, Rathore FA, Clegg D, et al. (2020) Pulmonary rehabilitation in COVID-19 patients: A scoping review of current practice and its application during the pandemic. Turk J Phys Med Rehab 66: 480-494.

13. Wang TJ, Chau B, Lui M, et al. (2020) Physical medicine and rehabilitation and pulmonary rehabilitation for COVID-19. Am J Phys Med Rehabil 99: 769-774.

DOI: $10.36959 / 719 / 580$

Copyright: (C) 2021 Abed FE. This is an open-access article distributed under the terms of the Creative Commons Attribution License, which permits unrestricted use, distribution, and reproduction in any medium, provided the original author and source are credited. 